\title{
Quand les difficultés du terrain sont ailleurs. Soubresauts d'un protocole de recherche sur la gauche révolutionnaire turque des années 1970
}

\author{
Paul Cormier \\ CUREJ, Université de Rouen, France
}

CONTACT :

13 rue de la Haie Vigné, 14000 Caen, France

paul.cormier01@gmail.com

\begin{abstract}
This article is a reflexive return to my doctoral field research on the biographical consequences of the revolutionary engagement in Turkey in the 1970s and has a twofold objective: first, it underlines that the difficulties of the research are not always where one expects them to be. Some preliminary concerns turn out to be unfounded, while other complications that are more difficult to anticipate emerge during the course of the survey and lead to adapt the initial project. Secondly, the article discusses the methodological combinations needed to investigate the research object when sources are scarce, fragmentary and involve historical distance.

\section{Résumé}

Cet article constitue un retour réflexif sur mon enquête de terrain doctorale sur les conséquences biographiques de l'engagement révolutionnaire au cours des années 1970 en Turquie et vise un double objectif : il souligne en premier lieu que les difficultés de l'enquête ne se situent pas toujours là où on les attend. Certaines inquiétudes liminaires se révèlent infondées tandis que d'autres complications plus difficiles à anticiper surgissent au cours de l'enquête et amènent à composer avec le projet initial. Dans un second temps, l'article revient sur les combinaisons méthodologiques nécessaires à l'investigation de l'objet de recherche quand les sources sont rares, fragmentaires et mettent en jeu la distance historique.
\end{abstract}

\section{Keymords}

Fieldwork, qualitative methods, biographical interviews, archives, leftists movements, Turkey.

\section{Mots clés}

Enquête de terrain, méthodes qualitatives, entretiens biographiques, archives, mouvements de gauche, Turquie.

La littérature en science politique consacrée aux questions méthodologiques et aux démêlés d'enquête de terrain dans des contextes étrangers, et moyen-orientaux en particulier, est aujourd'hui bien étayée. Ces travaux réflexifs se sont intéressés à la manière d'entrer sur un terrain (Vannetzel, 2010), de " gérer » son statut d'étranger (Massicard, 2002) ou encore à l'effet de dévoilement progressif de la «structuration » du terrain (Grojean, 2010). Ces deux derniers textes prennent d'ailleurs (au moins partiellement) le terrain turc pour cas d'étude. Parallèlement, les travaux sur les terrains «difficiles » (Boumaza et Campana, 2007) ont souligné les contraintes propres à l'espace enquêté (Bué, 2010) mais aussi celui que le contexte 
politique ou le régime politique peuvent faire peser sur l'enquêteur (Romani, 2007). La lecture de ces travaux, antérieurs à la réalisation de ma propre recherche doctorale, a largement contribué à structurer mes anticipations et appréhensions quant à sa réalisation. Si ces questionnements se sont retrouvés dans le déroulé de l'enquête - de manière nécessairement singulière - d'autres obstacles sont apparus progressivement que je n'avais guère anticipés. C'est, en premier lieu, sur cette surprise que s'interroge cet article. En d'autres termes, comment s'adapter quand les difficultés ne sont pas où elles semblaient être ? Pour déployer cette interrogation, je détaille certains épisodes du déroulé du terrain qui sont révélateurs de ce surgissement de contraintes et de la manière dont elles obligent à adapter son protocole de recherche sinon le cadrage même de l'enquête.

Comment rendre compte sociologiquement des conséquences biographiques de l'engagement politique en contexte autoritaire et répressif ? C'est à cette question que ma thèse de doctorat s'est efforcée de répondre à partir du cas des militant-es de la gauche révolutionnaire turque des années 1970. La recherche est basée sur l'hypothèse centrale selon laquelle les conséquences biographiques de la répression, en particulier après le coup d'État militaire de septembre 1980, sont différenciées pour les militant-es en fonction d'un ensemble de facteurs et de propriétés. Cependant, le projet de la thèse va au-delà de cette seule question. Il articule cette sociologie des parcours biographiques (au niveau micro) à l'évolution des organisations politiques de gauche (au niveau méso) et à la sociologie du régime politique turc (au niveau macro) depuis les années 1970. Revisitant la notion de carrière militante, ce travail propose, à partir d'une approche à la fois contextualiste et dispositionnaliste, une analyse dense des trajectoires individuelles à travers la notion d'économie des sphères de vie (professionnelle/scolaire, familiale et politique) attentive aux inscriptions sociales plurielles des acteurs. La réflexion porte sur les dynamiques et les temporalités des reconversions successives liées à un engagement radical dans un environnement autoritaire et répressif dans les deux principales villes du pays : Istanbul et Ankara. Mon approche est comparative sur le plan géographique tout en étant attentive aux configurations locales.

L'enquête de terrain s'est déroulée de 2013 à 2015 en Turquie et en France. Pour mener à bien cette enquête qui privilégie les «jeux d'échelles » (Revel, 1998), la thèse repose sur la combinaison de plusieurs sources complémentaires. La source principale est constituée d'une quarantaine d'entretiens biographiques avec d'ancien-nes militant-es de la gauche turque. Elle s'appuie également sur l'exploration de dix-huit textes de mémoires publiés par d'ancien-nes militant-es décrivant leur trajectoire. Une analyse de la presse turque et française complète le corpus. Enfin, des observations sur les mobilisations mémorielles contemporaines de la gauche ont également été réalisées. Il importe de souligner la dynamique d'entremêlement constant entre la construction de l'objet, la réalisation de l'enquête - et en particulier la présence sur le terrain - et le choix des méthodes utilisées ${ }^{1}$.

Ce retour réflexif sur mon enquête de terrain s'articule donc autour de ces deux principaux axes : dans un premier temps, je reviendrai sur les difficultés de l'enquête qui ne se trouvent pas toujours où on les attend, avant, dans un second temps, de détailler les combinaisons de sources adoptées dans le cours de l'enquête suivant l'évolution de l'objet de recherche et les difficultés rencontrées. Mais je commencerai par rappeler les principaux apports de ma thèse.

\footnotetext{
${ }^{1}$ Une dernière méthode a été mobilisée dans le cadre de cette recherche : l'analyse cartographique du militantisme à Istanbul et Ankara au cours des années 1970. N'intéressant pas directement l'objet de cet article, et déjà présentée et discutée par ailleurs (Cormier, 2019), elle ne fera pas l'objet d'une présentation systématique ici.
} 


\section{Etudier les conséquences biographiques de l'engagement en contexte autoritaire : hypothèses et résultats}

Plusieurs recherches se sont déjà intéressées aux conséquences biographiques de l'engagement, qu'il s'agisse du mouvement des droits civiques aux Etats-Unis (McAdam, 2012) ou encore de Mai 68 en France (Pagis, 2014 ; Fillieule et alii., 2018). Mais il s'agit à chaque fois de pays à la fois occidentaux et démocratiques. Cette recherche (Cormier, 2016a) déplace doublement le regard en mettant à l'épreuve les concepts forgés par la sociologie, et par la sociologie des mouvements sociaux en particulier, sur un cas d'étude, la Turquie, hors de l'Europe de l'Ouest et de l'Amérique du Nord et qui a connu une succession de régimes plus ou moins autoritaires au cours du XXe siècle. Ces deux caractéristiques, pourtant centrales pour la compréhension des mouvements sociaux, sont encore insuffisamment présentes dans les travaux de sociologie politique (Cormier, 2016b). Ce déplacement s'avère pourtant productif car il permet, en se demandant quelles sont les conséquences de l'engagement contestataire face à un régime autoritaire et répressif, de (re)poser une question centrale dans les sciences sociales : comment les évènements historiques pèsent-ils sur les comportements individuels ?

\section{La Turquie depuis le début des années 1970 : contextualisation de l'enquête}

Au cours de la décennie 1970, la Turquie, comme nombre de pays, connaît une importante vague de contestation politique sur fond de crise économique, politique et sociale. Le conflit oppose un mouvement de gauche protéiforme (mouvement syndical alors en plein développement, partis politiques socialistes et groupes d'origine étudiante ${ }^{2}$ tentés par l'action violente) à une extrême-droite milicienne et fortement structurée autour d'un parti, le Milliyetçi Hareket Partisi (MHP, Parti de l'action nationaliste) soutenu par les forces politiques conservatrices. Les affrontements de plus en plus violents entre militant-es de gauche et de droite font plus de 5000 victimes entre 1975 et 1980 sur l'ensemble du territoire (Gourisse, 2014). Les militant-es de la gauche révolutionnaire, initialement actif-ves sur les campus travaillent à s'implanter dans les lycées, les quartiers périphériques des grandes villes et, dans une moindre mesure, dans les campagnes. L'ampleur des mobilisations, l'intensité des luttes sociales et le foisonnement des initiatives locales contribuent, au-delà de la violence politique, à faire de cette période aujourd'hui stigmatisée en Turquie une période à la fois passionnante et méconnue. Jusqu'à la proclamation de l'état de siège dans la majorité des départements en décembre 1979, l'État turc parvient difficilement à maintenir l'ordre et à mettre un terme aux dynamiques de radicalisation politique à l'œuvre.

Cette décennie de fortes tensions politiques se clôt avec le coup d'État militaire du 12 septembre 1980 qui réprime durement la gauche ${ }^{3}$. La junte installe un régime militaire jusqu'en décembre 1983 et reconfigure en profondeur le pays : rédaction d'une nouvelle constitution restreignant drastiquement les libertés et installant l'armée au cœur des institutions, accélération de la libéralisation du pays sous l'égide du FMI et mise en œuvre d'une nouvelle doctrine d'Etat - la synthèse turco-islamique - alliant références religieuses et nationalisme intransigeant. Ce nouveau cadre autoritaire, que Gilles Dorronsoro, a qualifié de « régime sécuritaire » (Dorronsoro, 2005), criminalise toute dissidence politique au nom de la sécurité nationale, par ailleurs mise à mal par la guérilla kurde du PKK lancée en 1984. La gauche et ses militant-es,

\footnotetext{
${ }^{2}$ Ce sont ces groupes et militants qui sont au cour de l'enquête. On peut citer, entre autres, Devrimci Yol (Voie révolutionnaire), Devrimci Sol (Gauche révolutionnaire), Kurtuluş (Libération) ou encore Halkın Kurtuluşu (Libération du peuple).

${ }^{3}$ On estime en effet qu'environ 650000 personnes ont été arrêtées tandis que 49 ont été exécutées après condamnation à mort, des centaines sont décédées dans des conditions suspectes et près de 30000 ont été contraintes à l'exil (Massicard, 2012).
} 
durement touchés par la répression, politiquement délégitimés et interdits d'expression officielle jusqu'au mitan des années 1990, parviennent cependant à survivre en investissant les interstices du nouveau régime. L'arrivée au pouvoir de l'AKP en 2002 a semblé marquer une période d'ouverture et d'éloignement du militarisme du pays avant que l'autoritarisme, dans la foulée du mouvement Gezi de 2013 et de la tentative de coup d'Etat en juillet 2016, ne gouverne de nouveau les pratiques politiques.

S'inspirant des recherches de Bernard Lahire (Lahire, 2001, 2012) qu'elle applique au militantisme en contexte répressif, cette thèse met en œuvre une analyse des biographies attentive d'abord aux socialisations multiples, pourvoyeuses de dispositions et de capitaux. Une telle approche permet de rendre compte de manière fine des trajectoires différenciés d'entrée dans le militantisme révolutionnaire au cours des années 1970 en fonction des expériences vécues mais aussi de variables multiples telles la classe d'origine, le genre, l'origine ethnique, religieuse ou géographique et la transmission des préférences politique familiales. L'enquête fait ici ressortir la diversité des parcours militants au sein des groupes de la gauche révolutionnaire de la période et permet de distinguer trois micro-cohortes tant en termes d'âge que d'origine sociale : la première se compose des militant-es investi-es dans le militantisme de gauche à l'université dès la fin de la décennie 1960 et issu-es de milieux plus urbains et favorisés. La seconde est constituée par des individus originaire de province et de milieu rural qui sont lycéen-nes au tournant des années 1970 et qui deviennent étudiant-es au cours de cette décennie. Enfin, à mesure que les groupes de gauche s'implantent dans les quartiers périphériques au cours de la décennie 1970, se détache une troisième cohorte composée de lycéen-nes issu-es de familles souvent récemment installées dans les métropoles. Cette diversité dans les trajectoires initiales constitue à la fois un résultat probant de cette recherche permettant de mieux dessiner le paysage sociologique de la gauche révolutionnaire turque mais aussi le point d'appui à une analyse des conséquences biographiques de l'engagement face à sa répression.

Ce travail tient ensuite compte des inscriptions synchroniques plurielles des acteurs que l'on peut nommer sphères de vie (Passy, 1998). Il est possible d'en distinguer trois pour la clarté de l'analyse ${ }^{4}$ : la sphère familiale/privée, la sphère scolaire/professionnelle et la sphère militante. Ces trois sphères de vie sont en interaction permanente et plus ou moins en tension les unes avec les autres au sein de ce que l'on peut qualifier d' " économie des sphères de vie » (Passy, 2005). La prise en compte de la synchronie des inscriptions sociales des acteurs s'avère en effet nécessaire pour rendre compte des arbitrages rendus, des bifurcations ou des ruptures observables dans le cours des trajectoires. Il est ainsi possible de dégager des propositions générales à partir du cas turc : l'entrée, et surtout le maintien dans l'engagement radical - en l'occurrence révolutionnaire -, sont conditionnés par leur articulation de manière congruente aux autres sphères de vie. Plus la sphère militante est liée aux deux autres, plus l'engagement politique de l'individu est stable. Ainsi, au cours des années 1970, l'ensemble des activités des militant-es tend progressivement à s'organiser autour de leur engagement politique qui structure leur investissement scolaire et l'ensemble de leurs loisirs, de leur sociabilité et même de leurs amours. Mais les sphères restent toujours plus ou moins en tension : le militantisme révolutionnaire peut interférer avec la carrière scolaire (ou professionnelle) et l'environnement familial. Fragiles et non linéaires, ces engagements gagnent à être à également envisagés dans les effets qu'ils produisent à court et long terme. Après avoir approché les parcours des femmes et des hommes qui s'investissent dans le militantisme révolutionnaire au cours de la décennie 1970, cette perspective analytique permet également de rendre compte des effets multiples,

\footnotetext{
${ }^{4}$ Cette distinction est faite dans un but analytique. Les sphères de vie sont très largement imbriquées dans le cours ordinaire des existences.
} 
concomitants ou différés, de cet engagement dans les sphères professionnelles, familiales et militantes. La réflexion sur les conséquences biographiques de l'engagement contestataire en contexte autoritaire permet d'identifier des scénarios de déclassement et de reclassement social, liés à la durée ou à l'intensité des investissements militants. Elle offre ainsi une compréhension précise des logiques de reconversion des acteurs confrontés à la répression et à ses conséquences (exil, détention, clandestinité) et devant trouver à s'inscrire dans des espaces sociaux (professionnels, militants) eux-mêmes bouleversés et reconfigurés par le régime militaire et ses suites. Les trajectoires biographiques montrent en effet des reconversions difficiles tant dans le domaine professionnel (études interrompues, carrières hachées, déclassement professionnel) que personnel (entrée tardive dans des rôles sociaux liés au vieillissement tels que mariage ou parentalité) qui génèrent des désajustements sociaux.

On ne peut toutefois comprendre les logiques de l'engagement et leurs effets qu'en articulant les biographies ainsi conçues avec les effets et les transformations des différents contextes dans lesquels elles s'inscrivent. Ces derniers doivent cependant être approchés de manière précise comme je le montrerai dans la suite de cet article, au risque de relever de l'incantation. Cette recherche s'attache dès lors à distinguer ces contextes en recourant à la notion d'échelle. A l'échelle macro-sociologique, on saisit les dynamiques qu'impriment les mouvements sociaux sur le contexte politique, notamment au cours des années 1970. Ils contribuent à la politisation de certaines franges de la population (ouvriers, paysans, étudiants), entretenant un climat de revendications sociales et politiques allant des droits syndicaux à la reconnaissance des minorités, à commencer par les Kurdes. A partir des années 1980, les mobilisations en partie issues de la gauche des années 1970 maintiendront, quoique sans la même audience, ces revendications tout en s'impliquant dans d'autres causes (écologie, féminisme, altermondialisme). C'est sur cette conflictualité sociale initiée au cours des années 1960 et 1970 que s'appuient les militaires pour renverser le régime parlementaire en septembre 1980 et imposer un retour de bâton conservateur. Les changements politiques introduits par la junte (répression à grande échelle, nouvelle constitution) contraignent fortement les mobilisations et déterminent en partie leur faible capacité à se structurer depuis les années 1980. A l'échelle méso-sociologique, on distingue les différents espaces sociaux dans lesquels sont plongés les individus et qui produisent sur eux des effets tout en se transformant sous l'effet conjoint des dynamiques macro-sociales et des comportements individuels. Le mouvement de gauche des années 1970, dynamique et protéiforme, est ici envisagé à partir des groupes révolutionnaires multiples et concurrents constitués par les étudiant-es et lycéen-nes dans leurs établissements scolaires respectifs. L'analyse des entretiens et des sources collectées durant l'enquête permet de reconstituer la structuration (scissiparité forte, ressources limitées) et les transformations de la gauche révolutionnaire turque depuis les années 1970. Un autre résultat important de ce travail au niveau méso-sociologique est la mise en avant des recompositions de la gauche turque à partir des années 1980. La réapparition de diverses mobilisations au cours des années 1980 que l'on a souvent pu interpréter comme le signe de la naissance d'une « société civile » indépendante de l'Etat autant que du logiciel idéologique de l'extrême gauche (abandon du discours de la lutte des classes au profit de luttes dites identitaires) apparaît comme inscrite dans des continuités plutôt que dans une rupture avec les mobilisations des décennies antérieures. Autrement dit, en retissant ensemble les mobilisations collectives des années 1970 aux années 2000, on propose de déplacer le questionnement en observant comment le foisonnement associatif, a priori distancié de la politique, a opéré comme un vecteur de maintien des mobilisations de gauche en Turquie. L'apparente disparition de la gauche masque en réalité une mise en sommeil des registres et des répertoires de la gauche révolutionnaire (Osa, 2003).

A partir de ces éléments d'interprétation éclairés par l'analyse des échelles méso et macro, on peut dès lors questionner, au niveau individuel, la manière dont les savoir-faire, 
dispositions, réseaux issus des diverses expériences (militantes, scolaires etc.) sont réinvestis dans des pratiques professionnelles, des ambitions sociales ou d'autres engagements. Plus précisément, il s'agit ici de déplacer le regard vers d'éventuelles transformations des manières de porter de nouveaux problèmes dans l'espace public, de redéployer des dispositions acquises dans le militantisme sur d'autres répertoires d'actions, d'autres causes, dans un environnement politique, social et idéologique profondément transformé à partir des années 1980. L'étude des parcours militants montre ainsi la grande diversité d'espaces dans lesquels les acteurs se sont investis pendant ou après le régime militaire tant sur le plan professionnel que politique. J'ai souvent constaté l'imbrication entre les deux dimensions (professionnelle et politique) à travers la professionnalisation dans des emplois municipaux tenus par le parti social-démocrate ou dans le syndicalisme de branche. J'ai ainsi pu recomposer, au gré des entretiens, les différents espaces ayant servi de refuge aux militant-es gauche au fil des années (associations culturelles, défense des droits de l'Homme, syndicats, partis politiques, associations mémorielles...) et qui (re)composent au final un champ multi-organisationnel (Curtis et Zurcher, 1973) de gauche. La découverte de l'ensemble de ces espaces s'est faite au fil de l'enquête qui, sans prétendre les avoir tous épuisés, en dresse une cartographie et une chronologie les plus précises possibles en ce qui concerne les deux villes au cœur de ce travail. Au final, ce travail approfondit l'étude des interactions entre les transformations du régime turc et sa contestation dans une histoire longue en montrant comment une contestation du pouvoir est toujours parvenue à subsister, en dépit de la répression. Il souligne à cet égard que l'islam politique, aujourd'hui triomphant, n'a pas toujours été le seul ni le plus puissant adversaire d'un régime autoritaire au sein duquel l'armée joue un rôle central.

\section{Quand l'ordinaire supplante l'extraordinaire}

Avant de débuter, une enquête de terrain fait l'objet d'anticipations, en particulier pour tout jeune chercheur. On projette les difficultés et les succès escomptés. En l'occurrence, les difficultés ne se sont pas manifestées là où je les attendais. Je m'attarde ici sur deux exemples de difficultés apparues au cours de ma présence sur le terrain, alors même que celles j'escomptais ne s'avéraient pas déterminantes.

\section{Un terrain « difficile »? Anticipation(s) et réalité(s) de l'enquête de terrain}

Compte tenu du contexte politique turc, j'appréhendais un «terrain difficile » (Boumaza et Campana, 2007), en l'occurrence fortement contraint par une pression répressive sensible dans d'autres terrains autoritaires et auxquels plusieurs de mes collègues enquêtant notamment dans le monde arabe ont pu être confrontés au même moment. Or, sur ce plan, la réalisation de l'enquête de terrain s'est avérée moins difficile qu'escompté. Le plus difficile n'était donc pas la gestion d'événements extraordinaires comme une arrestation mais bien plutôt l'ordinaire de toute enquête et de toute construction d'une analyse scientifique (entrée sur le terrain, cadrage du projet, collecte des matériaux).

Enquêter sur un pays étranger oblige à anticiper et organiser la présence sur place de manière serrée dans la mesure où ce dernier ne m'était pas aisément accessible et qu'il m'était difficile de m'y inscrire de manière permanente. Ne résidant pas en Turquie au moment de l'enquête, celle-ci s'est déroulée en quatre phases ${ }^{5}$ : la première de la mi-juillet à la fin novembre 2013, la seconde au printemps 2014 (mai-juin), la troisième pendant tout le mois de novembre 2014 et la dernière entre avril et mai 2015. Le premier séjour, à Istanbul et Ankara,

\footnotetext{
${ }^{5}$ Ces quatre périodes de présence en Turquie et leur durée n'étaient pas initialement préméditées. Si je comptais y séjourner à plusieurs reprises, ce sont le déroulement et les besoins de l'enquête d'une part et les arbitrages personnels d'autre part qui ont imposé le calendrier.
} 
m'a permis de me familiariser avec le terrain, de prendre des contacts et de réaliser les premiers entretiens tout en accumulant de nombreuses données (archives, documents, photographies, livres, observations ethnographiques). Les deux terrains suivants ont été centrés sur Ankara où j'ai réalisé tous les entretiens prévus et poursuivi l'accumulation de données tout en réalisant des observations ethnographiques plus poussées lors des actions militantes auxquelles j'ai été convié. Le dernier moment, le plus court, a visé au même objectif sur Istanbul. Des contacts ont été entretenus à distance avec plusieurs enqueté-es entre chaque phase de terrain ce qui m'a permis de conserver un lien avec les milieux enquêtés mais aussi de préparer les différentes phases de terrain en les informant de mes venues en Turquie.

Bien plus que l'attitude des enquêté-es, je craignais initialement l'attitude des autorités turques à l'endroit de ma recherche. Le glissement autoritaire du régime et sa criminalisation des mobilisations protestataires depuis le mouvement Gezi de l'été 2013 - auquel la gauche turque avait été fortement associée - m'inquiétaient quant à ma capacité à mener à bien mon enquête. La féroce répression du mouvement Gezi à Taksim quelques semaines avant mon arrivée en Turquie a fait sept morts et une étudiante française arrêtée par la police pendant une manifestation a été détenue par les autorités avant d'être expulsée vers la France et interdite de séjour en Turquie. Sans vouloir surestimer la capacité subversive des travaux sociologiques ni l'omniscience de la police turque, ma situation était potentiellement fragile. Cette crainte était particulièrement forte lors des audiences du procès de la junte militaire au palais de justice de la capitale ou lorsque j'accompagnais les militant-es de la fédération des 78 'ards lors des manifestations de commémoration du coup d'Etat du 12 septembre 1980 devant l'ambassade des Etats-Unis à Ankara. J'ai eu un mouvement de recul assez net quand, à l'issue de la manifestation débouchant sur l'ambassade américaine - les militant-es voulaient y déposer une gerbe noire commémorant le soutien américain à la junte -, un mur de policiers armés et épaulés par des véhicules anti-émeute nous faisaient face. La manifestation s'est terminée et dispersée dans le calme. Je craignais une arrestation (qui m'aurait probablement valu une expulsion du territoire condamnant par conséquent l'enquête) lors de ma participation à ces diverses activités militantes et les questions des autorités sur les motifs de mon séjour, sur la nature de mon sujet et sur les contacts que j'avais dans le pays. Certain-es militant-es rencontré-es au cours de la thèse ont été condamné-es pour terrorisme et/ou activités clandestines et illégales en raison de leur militantisme passé. Par ailleurs, nombre d'entre eux/elles affichent publiquement leur soutien à la cause kurde ce qui les expose à l'accusation de soutien à une organisation terroriste, le PKK en l'occurrence.

Cependant, cette crainte ne s'est pas matérialisée. Si elle n'était peut-être pas totalement infondée, je n'ai pas senti de pression particulière, ni été arrêté, surveillé ou suivi pendant mon enquête. Les dates des périodes durant lesquelles j'étais présent sur le terrain ne sont pas sans signification dans la mesure où l'enquête précède la tentative de coup d'Etat de 2016 qui, par la réponse répressive apportée par le régime, aurait sans doute rendu considérablement plus difficile mon projet. La faible publicité et la faiblesse numérique des mouvements « d'anciens » de la gauche des années 1970 ne suscite guère la crainte et la surveillance des autorités. Mon enquête n'a donc pas été contrainte sur ce plan, ce qui ne signifie pas qu'aucune contrainte ne pesait sur mes enquêté-es. Un attentat revendiqué par l'Etat islamique devant la gare d'Ankara le 10 octobre 2015 (102 morts, plus de 500 blessés) a touché une manifestation de gauche - à laquelle participaient plusieurs de mes enquêté-es - quelques mois seulement après mon dernier séjour dans la capitale. Au final, mes inquiétudes, légitimes chez tout chercheur arrivant sur le terrain, se sont révélées partiellement infondées. Mon enquête n'a pas été entravée par les autorités et l'accueil que m'ont réservé les enquêté-es a dissipé mes appréhensions. Les difficultés sont davantage venues de la mise en œuvre du projet de recherche tel qu'il était initialement défini. 


\section{Un échec de comparaison}

Le projet de thèse s'appuyait sur un mémoire de Master consacré à la gauche révolutionnaire turque des années 1970. J'avais envisagé, pour ma recherche doctorale, d'élargir le projet en ajoutant les deux autres principales mouvances politiques qui investissent les campus turcs au cours de cette décennie : l'extrême droite réunie autour du MHP et l'islam politique. Les trois orientations politiques connaissent en effet un essor important et concomitant dans les années 1970 avant de connaître des destins contrastés. La gauche est fortement réprimée par le régime militaire (1980-1983) et peine à s'en relever tandis que l'extrême droite et surtout l'islam politique connaissent une ascension politique notable au point d'accéder au pouvoir. Recep Tayyip Erdoğan (né en 1954), actuel président de la République ou encore Devlet Bahçeli (né en 1948), secrétaire général du MHP depuis 2000, sont des enfants de cette décennie tourmentée sur laquelle s'interroge mon travail de thèse.

Gardant l'idée de partir du dispositif de recherche décrit plus haut, le projet était, à travers cette comparaison, $d$ 'analyser la manière dont les transformations du régime politique turc - auxquels certains de ces acteurs participent eux-mêmes - influencent le devenir politique de certaines mouvances et des militant-es qui s'y investissent. Il me fallait parvenir à enquêter au sein des deux autres univers politiques que je connaissais moins. Je décidais de commencer par l'islam politique. Sur les conseils de collègues, l'entrée sur le terrain a été abordée via un think tank politique ankariote proche du Parti de la justice et du développement [AKP, Adalet ve Kalkınma Partisi]. Elle s'est toutefois révélée assez pénible et la conduite de l'enquête l'a été tout autant. La méfiance des «gate keepers» (Broadhead et Rist, 1976), les interrogatoires multiples subis pour obtenir des entretiens et le conditionnement de l'accès au terrain par l'abandon de la posture comparative avec la gauche ont rendu le projet difficile à mettre en œuvre. Par ailleurs, les expériences d'enquête sur l'extrême droite turque ayant souligné la réticence du milieu à l'égard des chercheurs (Gourisse, 2011 : 85-86), j'ai préféré abandonner cette perspective qui, en plus de s'avérer complexe, était trop ambitieuse et difficilement réalisable dans le temps qui m'était imparti ${ }^{6}$. Je suis alors revenu sur l'idée de ne garder que l'étude de la gauche révolutionnaire dans le spectre de l'enquête.

Par ailleurs, tenter de pénétrer sur ces trois terrains particuliers et antagonistes de manière simultanée m'a révélé des difficultés et imposé des postures contradictoires. J'avais initialement choisi de cloisonner temporellement mon terrain d'enquête par commodité et par souci de cohérence et afin d'éviter tout conflit et situation de porte à faux entre les différents espaces investis, anticipant ainsi les possibles réticences face à au projet d'une comparaison entre mouvances politiques opposées. Le souci de maintenir l'étanchéité des terrains, par l'organisation de séjours séparés en Turquie, chacun visant à l'analyse d'une mouvance, me semblait ainsi a priori plus faisable qu'une comparaison concomitante. Des échanges avec des collègues m'ont convaincu d'abandonner cette méthode au profit d'une approche simultanée qui permet, en premier lieu, d'éviter un biais potentiel lié à un déséquilibre dans le traitement des terrains, le dernier se trouvant négligé en termes de temps consacré, et par l'impossibilité de revenir sur le terrain faute de temps si les données recueillies se révélaient insuffisantes. En second lieu, cette méthode permet d'appréhender simultanément la structuration et les codes des deux milieux et de confronter directement les modes d'accès au terrain.

Il n'est ainsi pas possible de prévenir toutes les difficultés qui surgissent au cours d'une enquête de terrain. Certaines, anticipées avant le début de l'enquête se révèlent infondées tandis que d'autres, considérées comme surmontables, peuvent perturber l'enquête au point d'imposer des retours en arrière ou des bifurcations comme ça a été le cas avec le projet de comparaison initial. Ces difficultés ne constituent pas tant des «ratés de terrain » (Stavo-Debauge, Roca i Escoda et Hummel, 2017) sur lesquels se penche volontiers la littérature, que des ratés

\footnotetext{
${ }^{6}$ «C'est un soulagement de ne plus me concentrer que sur la gauche », journal de thèse, 22/05/2014. J'ai tenu en parallèle un journal de thèse et un journal de terrain.
} 
d'anticipation et de conception du projet de recherche. Ces dernières sont sans doute autantsinon plus - à redouter que les premières. Il apparaît en effet particulièrement difficile de conduire, en Turquie comme ailleurs ${ }^{7}$, une recherche qui couvre par une collecte de données qualitatives (entretiens et observations) des militant-es de bords opposés sur un même terrain. En cela, le cas turc n'est pas si différent de terrains considérés comme moins «difficiles » $a$ priori. Les autres complications rencontrées se situent dans la collecte de données susceptibles d'étayer le projet d'analyse multiscalaire sur plusieurs décennies.

\section{Faire feu de toute source : la «triangulation » des matériaux d'enquête}

Pour mener à bien le projet d'étude des conséquences biographiques de l'engagement des militant-es de la gauche révolutionnaire dans la pluralité des contextes au sein desquels ils/elles ont été amenés à évoluer, le recours à une pluralité de méthodes s'est rapidement imposé. La principale méthode employée pour conduire cette recherche était celle des entretiens biographiques rétrospectifs. Ceux-ci constituent à la fois un outil central pour l'étude des trajectoires militantes mais offrent également un point de vue irremplaçable pour la reconstitution des espaces militants, qui plus est lorsque ceux-ci ont disparu, ainsi que sur les dispositifs répressifs auxquels les militant-es ont été confronté-es. La présence sur le terrain a révélé l'intérêt de travailler sur un deuxième support biographique : les mémoires militantes dont les publications se multiplient depuis le début des années 2000. Une autre méthode s'est ajoutée à ces matériaux. Les archives de presse et militantes encore disponibles ont été mobilisées pour la recomposition du contexte socio-politique général et la reconstitution des pratiques militantes. Cette «triangulation » est donc un effet de la présence sur le terrain et de la rareté des sources qui pousse à chercher tous les « indices » (Ginzburg, 1980) susceptibles d'éclairer l'enquête. Mais elle permet également de compenser les lacunes de chacune d'entre elles prise isolément tout en étant productrice d'une approche plus dense de la réalité étudiée.

\section{Travailler sur des entretiens biographiques rétrospectifs}

Une importante question méthodologique qui présidait à la préparation de l'enquête de terrain était la suivante : sur quels critères constituer le groupe d'enquêté-es ? En gardant à l'esprit que je ne pouvais travailler que sur des «restes de cohortes »(Offerlé, 1987: 75) du fait des désengagé-es et faute de représentativité possible, $\mathrm{j}$ 'ai focalisé mon attention sur les trois microcohortes mentionnées plus haut. Affinant le cadrage de l'enquête à mesure que le terrain avançait, je me suis concentré sur les militant-es actif-ves à Istanbul et Ankara dans des organisations révolutionnaires d'origine étudiante et les organisations de jeunesse des partis politiques. J'ai tenté, dans la mesure du possible, de me concentrer sur les militant-es « ordinaires » (Neveu, $2008: 308$ ), c'est-à-dire sur des personnes qui n'étaient pas forcément des cadres des organisations auxquelles elles appartenaient afin d'éviter le biais du survivant et une surreprésentation des leaders ${ }^{8}$. La majorité des enquêté-es ont été rencontré-es sur la base de la méthode dite de la « boule de neige » qui consiste à solliciter les enquêté-es sur d'éventuels contacts supplémentaires susceptibles de venir étoffer le travail. Cette méthode a l'avantage de garantir des recommandations auprès des personnes contactées mais le désavantage d'enfermer le chercheur dans certains réseaux, souvent locaux et socialement circonscrits. Si j'ai

\footnotetext{
${ }^{7}$ Sophie Duchesne (Duchesne, 2008: 707-708) avait discuté cette impossibilité à propos de l'étude des comportements électoraux dans sa recension de l'ouvrage de Céline Braconnier et Jean-Yves Dormagen, $L a$ démocratie de l'abstention.

${ }^{8}$ Toutefois, mon intégration aux réseaux militants passés ou restés en France m'a conduit à rencontrer plusieurs militant-es francophones dont les positions sociales et organisationnelles ne correspondaient pas nécessairement à celles de la majorité des militant-es des années 1970.
} 
rapidement obtenu la confiance des militant-es encore investis dans les organisations mémorielles ou politiques, il m'a fallu plus de patience pour accéder à d'autres profils, à commencer par les femmes. Une amie turque m'a permis d'en rencontrer deux que je n'aurais pu rencontrer à travers les réseaux militants très masculins au sein desquels je gravitais et où les femmes étaient très discrètes quand elles étaient présentes et refusaient poliment mes sollicitations, me renvoyant vers les hommes dominants de ces groupes. J'ai également essuyé un nombre important de refus d'entretien. Ceux-ci émanaient de profils très différents. Certaines personnes actuellement en poste (journalistes, universitaires) ont refusé des entretiens, même anonymisés, de peur d'évoquer un parcours qu'ils se sont efforcés de minimiser pour construire leur carrière. D'autres, pour qui les années 1970 et la répression constituent une période éprouvante sur laquelle ils ne souhaitent pas revenir, ont également refusé de s'entretenir avec moi. Un ancien militant m'a ainsi renvoyé vers son témoignage publié sans vouloir s'exprimer davantage. Mobilisant des motifs identiques, le dernier profil des refusant-es est constitué des militant-es installé-es en France suite à leur exil politique. Six personnes ainsi contactées n'ont pas donné suite à mes sollicitations, ont annulé nos rendezvous au dernier moment ou n'ont accepté qu'un entretien téléphonique. Sans doute est-ce là une nouvelle manifestation des difficultés du terrain qui, sans être propre à ce dernier, s'est présentée sans que je ne m'y attende vraiment; sas doute en raison de la durée relativement limitée de ma présence sur le terrain.

En ce qui concerne les entretiens, mon point d'entrée initial était la 78'liler Devrimci Federasyonu ${ }^{9}$ [Fédération révolutionnaire des soixante-dix-huitards] à Ankara. J'ai choisi ce point d'entrée car l'association était susceptible de concentrer un nombre important de militantes impliqué-es au sein de la gauche des années 1970 tout en me permettant d'observer les actions qu'elle mène. Si ce point d'entrée m'a offert la possibilité de rencontrer nombre d'enquêté-es, j'avais conscience que m'y cantonner m'aurait masqué les personnes qui n'en sont pas membres tout en risquant de m'enfermer au sein d'un seul réseau. Afin de contourner ces biais potentiels, j'ai décidé de multiplier les sites d'enquête (associations, syndicats...) à mesure qu'ils apparaissaient dans les entretiens ou dans la littérature afin d'élargir les réseaux potentiels ${ }^{10}$. Cette approche, qui aurait gagné à être approfondie par une présence prolongée sur le terrain, m'a permis de mettre en lumière la structuration de l'espace militant passé et présent de la gauche dans les deux villes enquêtées et les possibles reconversions qu'il a offertes tout en diversifiant les profils militants observés. Face au caractère aléatoire des rencontres et à la multiplication des refus d'entretien (une dizaine pour une quarantaine d'entretiens réalisés), j'avais projeté de resituer mon groupe d'enquêté-es au sein d'une population plus large via la réalisation d'une base prosopographique constituée à partir de notices biographiques rassemblées dans diverses publications sur la gauche turque. Celle-ci a été ébauchée dans le but de résumer certaines caractéristiques des trajectoires biographiques (données biographiques, scolaires, professionnelles, politiques) permettant de replacer et de comparer les enquêté-es interviewé-es dans un panel plus large, comme l'avaient fait certains travaux sur le militantisme en 1968 en France et même en Turquie (Baykam, 2002). Mais les données se sont - faute de sources - révélées trop éparses et incomplètes pour donner un résultat satisfaisant et une base de travail à la comparaison. Je l'ai donc abandonnée.

\footnotetext{
9 Fédération des révolutionnaires soixante-dix-huitards. Cette association d'ancien-nes militant-es de divers groupes révolutionnaires milite pour entretenir la mémoire des victimes de la répression sous le régime militaire et demande le jugement des militaires tortionnaires de la période.

${ }^{10}$ Ce point d'entrée n'a pas constitué un obstacle à la négociation d'entretiens avec des personnes qui n'en étaient pas membres en dépit du caractère concurrentiel de l'espace des mobilisations mémorielles de gauche. Les recommandations individuelles permises par la «boule de neige » permettaient le plus souvent de désamorcer les éventuelles suspicions.
} 
Les entretiens biographiques sous forme de récits de vie rétrospectifs ont été la principale méthode de travail mobilisée au cours de l'enquête. J'en ai conduit quarante-quatre avec d'ancien-nes militant-es de la gauche révolutionnaire turque mais aussi avec le fils d'un ancien militant et avec un militant trotskyste français présent en Turquie à l'époque. L'objectif de ces entretiens était double. Ils m'ont permis de recueillir le souvenir des événements passés (event history), leur datation, leur durées et leur succession dans les diverses sphères de vie (familiale, scolaire, professionnelle) ainsi que les ruptures et les transitions biographiques. Ils visent ensuite à saisir de la manière la plus détaillée possible le regard rétrospectif projeté par les militant-es sur leur parcours, le passé et le présent. Dès lors, les entretiens servent à rendre compte, de manière plus réflexive, des catégorisations, justifications et rationalisations utilisées par les acteurs pour expliquer leurs actions sans les rejeter au nom de «l'illusion biographique » (Bourdieu, 1986). Cette approche conduit à envisager le récit de vie comme le moment d'une trajectoire autant que comme le récit d'une trajectoire. En effet, bien souvent les entretiens sont l'occasion de raconter sa trajectoire biographique mais aussi - et dans certains cas surtout - de la relire eu égard à ce que l'on est devenu. Ainsi, Yiğit, engagé clandestinement au sein du TKP [Parti communiste de Turquie] alors qu'il est étudiant en médecine au cours des années 1970 a dû s'exiler en Europe après le coup d'Etat de septembre 1980. Il développe au cours de l'entretien un discours sur son propre parcours dans lequel il insiste sur la fragilité des destinées individuelles :

«[...] en l'espace de trois mois j'ai été élu secrétaire de cellule. Avec une formation... très légère. Et puis après je me suis retrouvé en exil et le parti m'a dit de rester en Europe, et je suis resté en Europe sur ordre du parti. Mais je veux dire... on était des gamins, on avait 22 ans, 20 ans, 19 ans bon ben voilà [il rit]. Et toute ma vie a changé à cause de ça. C'est... un truc très bête $»^{11}$.

A travers ces quelques phrases, on peut appréhender la manière dont les militant-es tentent de rationaliser leur biographie et les tensions qui la travaillent. D'un côté, le discours retisse ensemble les éléments d'une trajectoire militante brutalement interrompue par l'exil mais emprunte de fidélité à soi et au parti. De l'autre, il articule son vécu avec le poids des contraintes (le parti, le régime militaire) qui ont fait que « toute [s]a vie a changé ». La douleur du retour sur une trajectoire durablement affectée par ces ruptures est présentée sous l'angle d'un destin capricieux travaillé par le hasard («c'est très bête »). Ce propos n'est pas à éluder au nom de la reconstruction biographique mais au contraire à intégrer à l'analyse car il informe sur le regard que porte Yiğit sur sa propre trajectoire au moment de l'entretien. Mais l'entretien est aussi et souvent le moment d'un retour sur l'histoire de la gauche turque et d'une trajectoire collective vécue de manière plus ou moins douloureuse. Là encore, l'entretien avec Yiğit témoigne de cette dimension centrale des entretiens biographiques rétrospectifs :

«[...] comme il n’y a jamais eu de réflexion politique autocritique là-dessus [la violence politique des années 1970], même dans les groupes intellectuels, [...] personne n'a fait l'effort de dire 'Mais qu'est-ce qu'on a fait?' [...] Donc [...] ça s'est délité intellectuellement et politiquement et maintenant ce qui reste, ben vous voyez ce qui reste. C'est des petites chapelles qui reprennent exactement les mêmes trucs qu'avant. [Silence] Sans en tirer la moindre leçon $\gg^{12}$.

Cet extrait donne à voir selon quelles modalités et sur quels points d'articulation (absence d'autocritique, délitement intellectuel, sectarisme) se déploie un retour critique sur l'histoire de la gauche turque par un ancien militant mais vient aussi illustrer et justifier la distance que Yiğit

\footnotetext{
${ }^{11}$ Entretien avec Yiğit, Istanbul, 28/05/2015.

${ }^{12}$ Ibid.
} 
a mis entre ses ex-camarades et lui depuis ses années d'exil en France. Ainsi, les discours tenus en entretien doivent être rapportés aux expériences biographiques passées et aux positions sociales actuelles pour être intelligibles et exploités sociologiquement.

Les entretiens étaient cadrés par une grille d'entretien découpée en plusieurs sections qui articulaient chronologie et description des différentes sphères de vie (trajectoire familiale, scolaire/professionnelle, militante). Les entretiens se déroulant sous la forme d'entretiens semidirectifs, la grille était la plupart du temps adaptée suivant la tournure de la conversation et des retours en arrière réguliers pour préciser certains éléments étaient souvent effectués. Les entretiens ont été dans la grande majorité des cas conduits en turc, sauf avec les militant-es ayant exilés en France et qui se sont exprimés en anglais. La grille constituait davantage un cadre général qu'une liste de questions déroulées dans l'ordre. Je ne la mobilisais que sporadiquement et discrètement pour favoriser une discussion fluide. Celle-ci autorisait par conséquent des allers-retours dans le temps et dans les thématiques à mesure que des précisions étaient apportées ou sollicitées. J'ai privilégié des débuts d'entretien sur des thématiques plus facilement abordables (actions militantes actuelles, vie quotidienne...) pour privilégier l'abord de thématiques potentiellement plus personnelles (vie familiale, couple) ou douloureuses (expériences de répression) une fois certaines réticences tombées et la confiance instaurée.

Cependant, et non sans écho avec les nombreux refus, les entretiens n'ont pas toujours été aisés à conduire. Dérouté-es par les questions - pourtant annoncées - qui sortaient du strict cadre de leur militantisme, ils/elles étaient plus évasif-ves et tendu-es sur celles qui touchaient à leur vie professionnelle et personnelle. Le contexte politique de plus en plus autoritaire a sans conteste pesé dans certaines situations d'entretien. Les militant-es acceptaient en effet volontiers de parler de leur engagement passé et actuel qui était public - on a vu avec les refus qu'il en allait autrement pour les désengagés ou ceux/celles qui occupent des positions qui rendent plus difficile cette vocation - mais se révélaient plus réticent-es à livrer des informations privées susceptibles d'être dangereuses pour leurs proches. Les enquêté-es partageaient en cela les réserves des refusant-es. Si je rejoins Muriel Darmon lorsqu'elle écrit «que les modalités d'acceptation ou de refus [d'entretien] peuvent être rapportées à des positions dans l'espace des discours »(Darmon, 2005 : 107), j'ajouterais que ces mêmes modalités dépendent également, dans des contextes autoritaires, du risque qu'il y a à s'exprimer face au chercheur. Il m'a souvent fallu négocier et justifier l'importance de ces questions pour mieux comprendre les effets de leur engagement sur toutes les facettes de leurs trajectoires. Il est par ailleurs notable qu'hormis deux entretiens réalisés au domicile d'enquêté-es, tous se soient déroulés sur le lieu de travail des militant-es ${ }^{13}$, au siège de leur organisation militante ou dans un lieu public, souvent un café sans que je parvienne à négocier d'autres lieux alors que cette méthode est très critiquée dans les manuels de méthodologie (Barbot, 2010 : 123). Cela m'a privé d'un poste d'observation qui aurait pu se révéler potentiellement utile (lieu de vie, relations familiales) et a sans doute aussi rendu la parole intime plus difficile.

Les parcours biographiques ne sont pas toujours aisés à reconstituer, en particulier concernant la datation en raison des «infirmités de la mémoire » (Norton Cru, 2008 : 102). Il semble bien en effet pour les militant-es de la gauche turque qu'à l'instar des militant-es du Freedom Summer des années 1960, «les choses se passaient trop vite pour donner à leur mémoire le temps de fixer durablement des faits précis. Il y avait trop de choses à ressentir, à voir, à vivre » (McAdam, 2012 : 110). Pour contourner le «handicap de l'a posteriori » (Becker, 1987), j'avais initialement prévu de recourir aux calendriers de vie (Freedman et al., 1988 ; Fillieule et alii., 2018). Permettant de positionner les évènements biographiques centraux dans chacune des sphères de vie, ils sont un instrument d'objectivation des parcours biographiques mais également un outil de comparaison des trajectoires entre elles. Je n'ai

\footnotetext{
${ }^{13}$ On m'a très souvent indiqué l'adresse professionnelle des enquêté-es potentiel-les lors des entretiens ce qui explique que nombre de rencontres aient eu lieu dans ce cadre.
} 
toutefois pas réussi à systématiser le recours à cet outil méthodologique car les enquêté-es se sont montré-es démuni-es, sinon réticent-es, à les remplir au cours ou à l'issue de l'entretien, comme si leur administration "gâchait » la conversation. La méfiance répétée à l'encontre de cet outil - dont je n'ai sans doute pas su désamorcer la dimension intrusive - m'a conduit à l'abandonner. Face à ces réactions que je n'avais pas anticipées, j'ai mêlé dans l'analyse des entretiens une focalisation sur certaines étapes clés (entrée dans vie professionnelle, changement d'emploi, mariage, divorce, naissance des enfants, entrée/sortie de prison...) et des points de bifurcation dans le parcours, à la restitution d'une logique séquentielle qui ne correspond pas toujours à des moments précis et peut s'étirer selon des temporalités variables dans les différentes sphères de vie. Cette méthode soulève une seconde difficulté. En effet, les entretiens laissent parfois le chercheur devant un problème qui touche aux choix et aux enchaînements de séquences biographiques présentés selon une logique tantôt rationnelle tantôt contingente $^{14}$. Comment décider des "fragments ${ }^{15}$ à découper ? Ces choix s'opèrent lors de l'analyse du matériau et en lien avec la construction de l'argumentation scientifique. Le repérage de récurrences va permettre de qualifier une expérience collective et de donner de l'épaisseur à une somme de vécus singuliers tandis que certains épisodes idiosyncrasiques analysés en détails peuvent permettre d'identifier les logiques des ruptures biographiques individuelles. A titre d'exemple, au cours des années 1980, les prisonniers politiques de gauche sont régulièrement déplacés d'un établissement pénitentiaire à l'autre pour des raisons qui leur sont inconnues et qui relèvent des besoins de l'administration pénitentiaire qui doit gérer un afflux massif de détenus. Cette expérience de détention fragmentée, à la fois individuelle et collective, peut être appréhendée à travers les entretiens et les mémoires publiées après coup. Durdu Gevher relate dans ses souvenirs de prison être resté à la prison de Mamak (Ankara) pendant dix-huit mois avant d'être transféré à Metris (Istanbul) du jour au lendemain (Gevher, 2014 : 115). Teoman Ata est arrêté en 1981 pour appartenance au TKP. Dans un témoignage oral publié il raconte être resté un mois au DAL à Ankara avant d'être envoyé à Mamak de mai 1981 à juillet 1983 puis à Ulucanlar (Ankara), et à Bartin avant de retourner à Ulucanlar (TMMOB Mimarlar Odası Ankara Şubesi : 166-168). Au cours d'un entretien Şadi évoque sa propre trajectoire carcérale que j'ai pu mettre en regard des autres et ainsi mieux la recontextualiser :

«Il faut que tu sois à Istanbul pour participer au procès. Quand c'est fini tu n'es plus détenu tu es devenu prisonnier et on t'envoie dans d'autres villes. [...] Avant Metris c'était Selimiye [Istanbul]. Puis une autre qui s'appelle Davutpaşa près de Metris et finalement Metris. Mais après Metris on nous a envoyés dans une autre prison, non pas militaire, qui était à Çanakkale ${ }^{16}$.

Ces mouvements incessants d'une prison à l'autre ont pour but de déstructurer les réseaux militants et d'éloigner les prisonnier-ères de leurs réseaux sociaux. Ils ne seront pas sans effet à la sortie que ce soit en matière de trajectoire familiale ou en matière de retour à l'engagement militant. C'est pourquoi j'ai porté une attention particulière à leur analyse. La mise en valeur du matériau recueilli se joue dans ces allers-retours entre la question qui guide l'analyse et les données. Une fois encore, la contextualisation de la trajectoire et la comparaison avec les données objectives obtenues par ailleurs doivent permettre de démêler ou de nuancer (sans prétention à l'omniscience) les défauts de la mémoire et « l'illusion téléologique » (Schwartz, 1990 : 174) à l'œuvre. Par exemple, la consultation de la presse m'a permis de dater précisément

\footnotetext{
${ }^{14}$ Du type «j'ai toujours voulu faire ceci » ou, tout au contraire, «c'est le hasard qui a fait que... » (Grossetti : 2006, 26).

${ }^{15}$ Pour reprendre le terme turc, parça, employé par Hüseyin pour qualifier ses souvenirs de cette période.

${ }^{16}$ Entretien avec Şadi, Istanbul, 25/10/2013.
} 
des événements (manifestations, assassinats de militant-es) mentionnés dans les entretiens sans que l'enquêté-e ne parvienne à dater précisément l'événement. De la même manière la lecture des revues militantes des années 1970 - comme Dev Yol - m'a permis de resituer certaines pratiques militantes comme le contenu des séminaires de lecture dont les textes obligatoires étaient listés d'un numéro à l'autre.

\section{Croiser les sources biographiques: les mémoires militantes en regard des entretiens biographiques}

Les mémoires de militant-es sont, depuis les travaux pionniers de Bernard Pudal et Claude Pennetier, des sources instructives pour l'enquête. J'entends ici par mémoires des textes écrits par des militant-es impliqué-es dans les groupes révolutionnaires au cours des années 1970 et écrits à la première personne dans une perspective autobiographique. Les témoignages regroupent les textes écrits par des personnes non directement impliquées dans les organisations politiques. Ces témoignages peuvent être collectifs (Delikanlı et Delikanl1, 2014) ou individuels comme dans le cas du journaliste Hasan Cemal (Cemal, 2012). Les publications de mémoires militantes et de témoignages autour du 12/9/1980 se multiplient en Turquie depuis le milieu des années 2000 - bien que des textes aient été publiés auparavant - chez des éditeurs engagés à gauche (Ayrıntı, Imge, Nota Bene...). Leur existence et leur importance me sont apparues en fréquentant les librairies de Turquie dans lesquelles je cherchais des travaux historiques et sociologiques. J'ai par la suite exercé, tout au long de ma présence sur le terrain (2012-2015), une vigilance concernant les publications de ces textes. J'en ai analysé dix-huit issus de membres de différentes organisations, bien que celles des anciens cadres de Dev Yol soient largement dominantes quantitativement. Cette domination de la parole autorisée des cadres des groupes révolutionnaires m'a amené à pousser mes investigations vers les militant-es dont la parole n'était pas relayée et à mettre en regard leur parole recueillie en entretien avec les mémoires publiées. La situation d'enquête séparait donc les deux sources. Les enquêté-es avaient lesquel-les j'ai eu l'occasion de réaliser des entretiens n'écrivaient pas. Le corpus de textes mobilisé recouvre deux formes principales. La première, assez classique, est un récit chronologique à la première personne qui part généralement de l'enfance et qui va jusqu'à la fin des années 1970. La seconde compose des mémoires sous forme dialoguée. Ces deux types de textes contiennent souvent des photographies et, si l'auteur s'adonne à la poésie ce qui n'est pas rare, un choix de poèmes. Ils constituent un matériau très utile qui permet d'analyser sociologiquement les situations, les acteurs, les relations présentées tant sur la forme que sur le fond.

Reste que ces mémoires comportent, vis-à-vis de ma question de recherche, une série de lacunes. La première touche évidemment à la question de la reconstruction a posteriori déjà évoquée concernant les entretiens. La seconde est liée à la périodisation. En effet, les mémoires s'arrêtent généralement soit juste avant le coup d'Etat soit à la sortie de prison, contournant ainsi la question de l' « après ». Gün Zileli est un des seuls militants de mon corpus à avoir fait une autobiographie qui va au-delà de 1980, même s'il reste très discret sur les dimensions non directement politiques (familiale, professionnelle) de son parcours (Zileli, 2002, 2003). Ces textes contiennent également un biais important lié au fait qu'ils sont généralement publiés par les militant-es les plus doté-es en capitaux culturels et scolaires mais aussi en capital symbolique. Ils sont tous d'anciens cadres dirigeants de leurs groupes respectifs (Oğuzhan Müftüoğlu, Gün Zileli, Sedat Göçmen...). Comme l'écrit Catherine Leclercq, « s'en tenir à la production des mémorialistes reviendrait à avaliser le monopole narratif de ceux que leurs ressources sociales, leur capital personnel, leur sentiment de compétence, habilitent à l'egohistoire et autorisent à investir le champ éditorial : ce faisant on tendrait à survaloriser l'expérience des plus enclins à les faire voir et à la faire valoir » (Leclercq, $2005: 132$ ). Cet effet de domination est particulièrement criant en ce qui concerne les mémoires écrites par des 
femmes. Aucune des mémoires que j'ai pu consulter n'a été écrite par une femme militante. Les mémoires féminines du militantisme et de la répression sont exprimées dans des publications collectives également mobilisées (Akal, 2011 ; Collectif, 2011 ; Sağır, 2015). Il invite par ailleurs à questionner le rapport à l'écriture qu'entretiennent les militant-es. Bien que nombre d'entre eux/elles aient fait des études secondaires et supérieures, l'écriture, et en particulier l'écriture de soi, reste peu répandue et peu publicisée.

Comme dans le cas des entretiens ou des archives (cf. infra), ces biais potentiels peuvent être contournés de deux manières différentes. J'ai veillé à recouper les informations contenues dans ces textes avec les informations (dates, lieux, événements) contenues dans d'autres types de sources (archives militantes, sources de presse). Ensuite, comme il en va pour tout document autobiographique, j'ai approché les mémoires avec un regard critique attentif à l'expression des subjectivités intrinsèques à l'objet et des reconstructions a posteriori sans pour autant le rejeter d'emblée (Smith et Watson, 2010). Par exemple, Oğuzhan Müftüoğlu, cadre dirigeant de Dev Yol dans les années 1970, reste évasif dans ses mémoires quant à la situation subordonnée des femmes au sein des groupes révolutionnaires. La question de la domination masculine a été abordée à plusieurs reprises au cours des entretiens que j'ai menés et soulignée par des témoignages écrits (Akal, 2011). Müftüoğlu minimise de la même manière l'interdit posé sur les relations amoureuses et la distance imposée entre garçons et filles militant dans les groupes comme Dev Yol: « Nous en avons beaucoup parlé mais nous ne sommes pas parvenus à aboutir à une conclusion » (Müftüoğlu, 1989 : 217). Les entretiens menés avec d'autres militant-es ont pourtant confirmé les pressions exercées sur les militant-es en couple. Si cette ambivalence des dirigeants s'explique par ailleurs, elle n'en rappelle pas moins, sur le plan méthodologique, la nécessité de critiquer et de croiser les sources. Ces publications apportent quantité de détails sur le parcours de leur auteur mais également sur le fonctionnement des organisations, leurs relations, les lieux investis et les modalités de la répression. Mis en regard des archives des groupes, des archives de presse et des entretiens biographiques, ces mémoires permettent d'élargir le spectre des sources disponibles et favorisent les recoupements. D'autant qu'elles contiennent elles-mêmes des archives reproduites (photographies, affiches...), parfois personnelles, offrant ainsi de nouvelles données au chercheur. La question des archives constitue à cet égard une dimension importante dans le recueil du matériau de ce travail.

\section{Accéder aux archives}

Une des principales difficultés que ma perspective socio-historique a dû affronter a été l'absence d'archives ou, à tout le moins, leur éparpillement. Comment dès lors mener une enquête à dimension historique (presque) sans archives ? Il a fallu mettre en œuvre différentes techniques pour en trouver et parvenir à les exploiter. Mal connues et mal exploitées, les archives turques constituent, pour reprendre le mot de Michel Lesure, « un monde ignoré » (Lesure, 2013 : 13), et ce, à tous les sens du terme. Elles sont de plusieurs types.

Le premier bloc d'archives qui aurait notamment permis d'éclairer la période du régime militaire est composé par les archives d'Etat, et en particulier les archives militaires. Malheureusement classées, et a priori localisées au siège de l'état-major à Ankara, celles-ci ne sont pas ouvertes au public. Pour compenser ce problème, je me suis tourné vers la littérature de seconde main dite « grise » qui se compose de publications (rapports, ouvrages) émanant de l'institution militaire elle-même, des statistiques officielles et de rapports parlementaires et d'ONG et qui s'échelonnent dans le temps du régime militaire à aujourd'hui. Ces documents fournissent nombre d'informations utiles et mettent en forme les discours des protagonistes impliqués, à l'époque comme a posteriori, dans l'interprétation des évènements des années 1970, des « raisons » du coup d'Etat et de la répression sous le régime militaire. Ces données sont particulièrement utiles pour appréhender les transformations du régime turc après le coup 
d'Etat de septembre 1980: mise en œuvre de la répression, changements constitutionnels, options politiques et économiques du régime militaire...

Le second groupe d'archives est constitué des archives de presse que j'ai largement mobilisées. J'ai consulté plusieurs périodiques pour établir la chronologie des évènements et les discours alors tenus par les contemporains. Je me suis basé sur un dépouillement du quotidien Milliyet pour la période 1969-1980 en le confrontant aux chronologies présentes dans 1'Encyclopédie de la République (Cumhuriyet Ansiklopedisi, 2002). En dépit de leurs défauts (Fillieule, 2007), on obtient, à travers les sources de presse nombre d'informations d'ordre politique sur une longue période mais aussi sur les événements en lien avec le militantisme survenus dans les deux villes étudiées. J'ai également dépouillé, sur la période 1978-2014, le journal Le Monde en relevant les articles traitant de la Turquie ou des réfugiés turcs en France. Ce travail s'est avéré utile bien que partiel pour obtenir des informations sur la période du régime militaire au cours de laquelle la presse turque censurée se révèle fragile. Pour la période plus récente (2013-2016), j'ai mobilisé le quotidien Cumhuriyet [République], plus enclin à rapporter les mobilisations de gauche que les autres. Ces deux premiers groupes de données archivistiques ont été particulièrement utiles pour contextualiser les trajectoires militantes aux échelles macro et meso.

Le troisième et dernier bloc d'archives mobilisées concernait les archives des groupes militants passés et présents qui se trouvaient au cœur de l'enquête. Là encore, l'accès aux archives militantes des années 1970 n'a pas toujours été aisé. Certaines organisations, de nature clandestine, veillaient à ne pas produire d'archives internes. Seules subsistent les publications (revues, tracts, brochures) qu'elles publiaient alors et qui sont relativement accessibles, notamment en ce qui concerne les organisations les plus importantes (Dev-Yol, Dev-Sol, Kurtuluş...) ou les publications d'autojustification collective publiées a posteriori (Müftüoglu, 1989). J'ai eu accès aux archives des revues Devrimci Gençlik [Jeunesse révolutionnaire] et Devrimci Yol [Voie révolutionnaire] pour la période 1977-1980 suite à la leur mise en ligne par un collectif de jeunes chercheur-es turc-ques travaillant aux Etats-Unis ${ }^{17}$. Les autres publications militantes sont plus difficiles d'accès et plus éparpillées. En ce qui concerne les organisations légales (partis politiques, associations), la destruction préventive ou punitive des archives sous le régime militaire ${ }^{18}$ et les difficultés d'accès aux archives militaires m'ont rendu la tâche ardue. Si des associations turques comme la TÜSTAV ${ }^{19}$ travaillent à constituer des fonds d'archives sur la gauche turque, ils ne concernent guère la gauche révolutionnaire et se concentrent plutôt sur le syndicalisme ou les partis socialistes légaux. Le dernier moyen d'obtenir des archives a consisté à interroger systématiquement les enquêté-es sur leur possession éventuelle de documents, quel que soit leur type. Très peu disposaient de sources d'époque, la plupart les ayant détruites pour ne pas qu'elles soient saisies après le coup d'Etat et aient risqué de les compromettre. Seuls quelques-un-es les ont gardées, cachées ou confiées à la famille ${ }^{20}$ et ont pu me transmettre des publications et des photographies. Quant aux archives militantes contemporaines, elles conservent la trace de la tourmente et des pratiques politiques passées. La 78 'liler $D F$ ne dispose que de peu d'archives, y compris sur ses propres membres.

\footnotetext{
${ }^{17}$ URL : http://devrimciyolarsivi.org/

${ }^{18}$ A titre d'exemple, les Halk Evleri [Maisons du peuple], initialement fondées sous le régime kémaliste pour promouvoir le régime, constituaient un réseau de foyers implantés dans les quartiers des grandes villes et dans les campagnes. Très largement engagées à gauche depuis les années 1970, elles sont fermées par la junte en 1980 et ne rouvrent timidement qu'en 1987. L'armée détruit la grande majorité des archives saisies sous le régime militaire.

${ }^{19}$ Türkiye Sosyal Tarih Araștırma Vakfi [Fondation de recherche en histoire sociale de Turquie]. Créée en 1992, la TÜSTAV collecte, classe et met à disposition des chercheur-euses et des militant-es intéressé-es les archives de la gauche turque.

${ }^{20}$ Un enquêté a ainsi confié ses archives à un cousin militaire de confiance qui risquait peu de voir son logement soumis à une perquisition.
} 
La consultation de ces documents se révèle toutefois particulièrement riche pour saisir les discours tenus par les organisations révolutionnaires à travers leurs publications, leur rhétorique, leur positionnement politique et la culture militante qu'elles véhiculent.

Les travaux, sur d'autres terrains, consacrés au recours à l'archive d'organisations politiques (de Castro Rocha et Goirand, 2010) ont souligné plusieurs dimensions que l'on retrouve dans le cas des archives de la gauche révolutionnaire turque. L'accès aux archives était central pour recueillir des données de première main sur le fonctionnement des groupes révolutionnaires au cours des années 1970 et leurs conditions de survie et de recomposition après le régime militaire. Mais les archives, lacunaires, devaient être complétées par d'autres sources. Les entretiens et les mémoires militants (qui s'appuient pour une part sur des archives personnelles), constituent un complément central, à travers le détour de la mémoire, pour accéder au passé des groupes militants. Les entretiens permettent par ailleurs de saisir l'entremêlement des histoires individuelles et celles des groupes dans lesquelles elles se sont inscrites ainsi que les représentations qui circulent aujourd'hui, qu'elles soient singulières ou collectives. L'intérêt de ce recoupement est de saisir les transformations opérées par le vécu et la mémoire. $\mathrm{Ou}$, au contraire, de les mobiliser comme un outil d'interprétation, de décryptage de certaines archives ou de certains pans d'archives. On peut ainsi croiser les publications de photos des «martyrs » [şehit] tombés lors des affrontements entre militant-es révolutionnaires et d'extrême droite dans les revues militantes avec les récits, recueillis en entretien, des défilés chargés d'émotion qui accompagnaient les enterrements des militant-es décédé-es lors de ces affrontements. Ce croisement permet d'articuler le discours public des groupes politiques sur les événements et le vécu collectif sinon intime des militant-es. En second lieu, la difficulté d'accéder aux archives dans le cas turc rejoint les analyses consacrées au Parti des travailleurs brésilien qui « invitent à abandonner le fétichisme des sources écrites » (ibid. : 123) qui ne sont ni plus explicites ni plus accessibles (sinon moins) que les sources orales. La disparition, la fermeture de l'accès aux sources et leur disparité invitent en effet à la prudence et militent là encore pour la piste du croisement. De la même manière, elles ne sont pas plus «représentatives» que les entretiens et également chargées de «biais» (sélectivité, subjectivité) qu'il s'agit de contrôler par la vigilance méthodologique comme dans le cas des mémoires militantes. Ainsi, l'analyse des listes de lecture obligatoires diffusées dans les revues des différents groupes ne présage en rien de leur respect ou des conditions de leur appropriation par les cellules locales. En somme, si l'accès aux archives, de même que les entretiens, s'est fait au gré des aléas du terrain, des «trouvailles » et des relations établies avec les enquêté-es, l'impératif méthodologique demeure de contrôler les interprétations et les rapprochements opérés de manière à limiter les biais d'analyse.

\section{Deux exemples pour conclure}

Je présenterai, pour finir, deux exemples qui illustrent les éléments discutés dans cet article. En premier lieu, je montrerai la manière dont j'ai procédé pour rendre compte des conséquences biographiques de l'engagement, à la fois individuellement et collectivement, à partir des entretiens rétrospectifs. En second lieu, je montrerai comment s'opérationnalise le croisement des sources pour reconstituer des contextes politiques disparus.

La principale méthode de travail mobilisée au cours de l'enquête était le recueil d'entretiens biographiques sous forme de récits de vie rétrospectifs. A l'échelle individuelle, ils m'ont permis de recomposer les trajectoires biographiques de mes enquêté-es ainsi que les ruptures et les transitions biographiques auxquelles ils/elles ont été confronté-es. Au niveau collectif, il s'est agi de mettre en lien ces trajectoires pour dépasser les idiosyncrasies biographiques et rendre compte de la dimension collective de l'expérience militante et de ses conséquences. J'ai ainsi dégagé, ainsi que je le mentionnais au début de cet article, trois microcohortes qui correspondent à des vécus différents au cours de la décennie 1970 et après. La 
question des reconversions scolaires et professionnelles de la seconde cohorte, née entre 195152 et 1958-59 et composée de lycéen-nes au tournant des années 1970 qui deviennent étudiantes au cours de cette décennie avant d'être, pour la plupart, emprisonné-es au cours des années 1980, illustre bien cette situation. Les reconversions ne peuvent être saisies qu'en articulant les trajectoires passées des acteurs et les contraintes structurelles auxquelles ils font face (Denave, 2015). J'ai travaillé à mettre en valeur les trajectoires individuelles en cherchant à dégager les dispositions et les capitaux sur lesquels les acteurs-trices ont pu faire fond tout en montrant que ces dernières s'inscrivent dans une réalité collective. J'ai pu montrer que les reconversions reflètent les inégalités sociales initialement perceptibles dans la composition des groupes révolutionnaires : elles dépendent alors de la cohorte d'appartenance (et donc de l'âge) ainsi que des capitaux disponibles.

La situation financière dans laquelle se trouvent les militant-es est très précaire tout au long des années 1980. Beaucoup d'entre eux/elles ont vu leur trajectoire scolaire interrompue par le militantisme puis par la répression comme en témoigne Celalettin : «La dernière année d'université, la quatrième, je n'allais pas en cours parce que je militais et je risquais de me faire arrêter en allant à l'école. Du coup je n'ai pas fini mes études $»^{21}$. Nombreux-ses sont ceux/celles au sein de la seconde cohorte qui ont dû interrompre leurs études suite au coup d'Etat de 1980. Ils/elles sont alors soit lycéen-nes diplômé-es et ne parviennent pas à entrer à l'université comme Leyla et Özer, soit ils/elles sont déjà étudiant-es à l'université et sont contraints de la quitter. C'est le cas pour Hüsnü et Irfan. De son côté, Yiğit, étudiant en médecine à Ankara quitte la Turquie sur ordre de son parti (le TIP), abandonnant là ses études qu'il ne finit pas une fois arrivé en Belgique. Hüsniye, très désireuse d'entrer à l'université après ses études secondaires difficiles à Istanbul loin de sa famille est emprisonnée jusqu'en 1984. Libérée, elle tente d'entrer au conservatoire avec le soutien de sa famille pour faire de la musique ou du théâtre - activité qu'elle a découverte à travers le militantisme - mais elle n'est pas reçue. Elle cherche alors du travail et continue le théâtre dans des troupes syndicales amateures. Cette impossibilité de finir ou de commencer les études supérieures qui s'ouvraient devant eux alors qu'ils étaient parfois les premiers de leur famille à y avoir accès, constitue une grande souffrance chez les militant-es. Pris-es dans la nécessité de survivre à leur sortie de détention, ils abandonnent leur carrière scolaire faute de capitaux suffisants, pour entrer directement dans une carrière professionnelle. Certain-es reprendront toutefois des études quelques années plus tard, une fois leur situation stabilisée et le contexte plus propice. Ce projet leur permet également d'assurer un diplôme crucial (capital scolaire) dans les stratégies de reconversion tout en faisant le lien entre l'avant et l'après coup d'Etat dans un désir de revanche sur les autorités et de continuité de soi. Asiye, après avoir quitté Istanbul et l'Académie des Beaux-arts face à la répression pour se réfugier à Ankara, s'y réinscrit en littérature après avoir occupé quelques petits boulots pour vivre. Elle espère ainsi pouvoir décrocher un emploi stable et mieux rémunéré. Après avoir travaillé plusieurs années dans l'imprimerie de son oncle et s'être constitué un petit capital économique, Nejat K. reprend les études de droit qu'il avait commencées à Istanbul et que la prison avait interrompues. Il obtient finalement son diplôme d'avocat et il exerce jusqu'à sa retraite à Ankara. Fecire avait, pour sa part, obtenu une place en faculté de biologie quelques temps avant d'être incarcérée pendant deux ans en 1981 pour son appartenance à Dev-Yol. Elle se présente dès sa sortie au concours et décroche la faculté d'économie d'Ankara dont elle ressort diplômée. Les entretiens biographiques permettent donc à la fois une étude des parcours individuels tout en fournissant par leur mise en relation un outil d'intelligibilité des trajectoires collectives. Ils peuvent également appuyer le travail de reconstitution d'espaces sociaux disparus.

\footnotetext{
${ }^{21}$ Entretien avec Celalettin, Istanbul, 02/04/2009.
} 
La reconstitution des réalités sociales passées à partir de la «triangulation » des sources offre une meilleure intelligibilité des contextes dans lesquels les militant-es ont pu évoluer. J'ai par exemple eu recours à cette méthode pour mieux comprendre comment se concrétisait l'action des groupes révolutionnaires sur les campus. Au cours des années 1970, les organisations de gauche se mobilisent au sein des universités pour défendre les droits des étudiant-es. Elles sont à la fois reconnues par les administrations qui cherchent des moyens de limiter l'agitation et en même temps illégales et réprimées par les autorités. Les archives du groupe de Dev-Genç (qui devient par la suite $\mathrm{Dev}-\mathrm{Yol}$ ) montrent que le groupe se mobilise par exemple pour demander la création de tarifs étudiants dans les transports en commun stambouliotes (Dev-Genç, 1999 : 93), ce que plusieurs entretiens ont confirmé. Parallèlement, les groupes mettent en place des structures élues qui représentent les étudiants auprès de l'administration. Ces élections sont censées légitimer le pouvoir de l'organisation victorieuse dans les lieux où elle est majoritaire et sont utilisées pour marginaliser les autres. Les résultats des élections dans les différents établissements universitaires, mais aussi dans les conseils de yurt [cité universitaire] ${ }^{22}$, où l'organisation est implantée, sont publiés dans les revues militantes ${ }^{23}$ et permettent de saisir établissement par établissement les rapports de force entre groupes révolutionnaires et ainsi pouvoir cartographier leur implantation suivant les villes. Les entretiens viennent également compléter ces sources à partir de récits de pratiques, permettant ainsi d'affiner les éléments trouvés dans les archives. Ainsi, Ferhat, étudiant à ODTÜ [Orta Dogu Teknik Üniversitesi, Université technique du Moyen-Orient] à Ankara, fait partie du TKP et se trouve en minorité face à $\mathrm{Dev}-\mathrm{Yol}$ sur le campus :

« J'ai été élu représentant de la classe de management, de mon département [...]. Il y avait très peu d'élus de notre organisation, j'étais parmi les rares personnes. Dev-Yol avait le monopole à ODTÜ. [...]

P : Il y avait des élus de chaque département au sein de ÖTK [Öğrenci Temsilcileri Konseyi, Conseil représentatif des étudiants]?

F : Voilà, une sorte d'assemblée. Mais il y avait une autre branche, les élections de faculté. On se présentait en tant que liste. Donc ceux qui étaient de notre organisation et puis les sociaux-démocrates [...], on avait obtenu l'administration de cette association. [...] C'était très symbolique finalement. L'ÖTK, c'était Dev-Yol qui faisait tout. Je faisais des choses mais c'était pour... vraiment des questions techniques. En tant que représentant de la classe on se réunissait entre nous, on pouvait proposer des choses à ÖTK pour les faire accepter. Ça marchait jamais $»^{24}$

On voit donc, à travers l'exemple de l'organisation des élections étudiantes sur les campus universitaires, l'utilité de la triangulation des sources pour mettre en valeur des données initialement parcellaires mais aussi pour initier des jeux d'échelles allant des résultats globaux des élections étudiantes aux récits de pratiques localisées.

\section{Conclusion}

Toute enquête de sciences sociales génère des anticipations et des appréhensions quant à sa réalisation, a fortiori quand il s'agit d'enquêter à l'étranger sur des opposant-es à un régime de plus en plus autoritaire comme c'est le cas en Turquie. A travers le récit de mon enquête de terrain, j'ai voulu montrer que les difficultés ne se nichent pas toujours là où on les attend. Sans qu'il ne pèse directement sur le déroulé de mon enquête en termes de sécurité comme je le

\footnotetext{
${ }^{22}$ Dev-Yol, ${ }^{\circ} 28,05 / 1979$, pp. 6-7.

${ }^{23}$ Dev-Yol, $\mathrm{n}^{\circ} 18,22 / 05 / 1978$.

${ }^{24}$ Entretien avec Ferhat, Istanbul, 30/04/2015.
} 
prévoyais, le contexte politique s'est certes révélé être un obstacle dans l'accès aux sources, à commencer par les archives. De même, les refus d'entretien et les résistances des enquêté-es quant au fait de parler de leur vie privée révèlent, d'une part, des réticences à parler d'autre chose que de leur engagement et, d'autre part, des craintes de livrer des détails qui pourraient être utilisés contre eux ou contre leurs proches. Par ailleurs, le projet a dû être reconfiguré face à la difficulté d'ouvrir certaines portes, non du fait des autorités mais des milieux enquêtés euxmêmes. L'échec de la mise en place d'une comparaison entre militant-es de gauche, de droite et de l'islam politique m'a ainsi obligé à reconsidérer le projet initial au cadrage inadapté. Si je n'ignorais pas les difficultés d'une telle entreprise, j'ai néanmoins dû réorienter l'enquête sur la seule gauche révolutionnaire. On pourrait même avancer, au-delà de l'idée selon laquelle les difficultés de l'enquête ne se nichent pas toujours où on les attend, que travailler en terrain autoritaire ne doit pas amener à accorder une attention trop grande aux « situations limites » (Céfaï et Amiraux, 2002) ou au contournement de la censure et de la surveillance (Fournier, 1996) en sous-estimant ainsi les difficultés sociologiques « ordinaires » communes à tous les objets de recherche.

La seconde difficulté qui s'est présentée, liée au fait de travailler sur un épisode historique avec des sources parcellaires, a été celle de la collecte des données et de leur interprétation pour étayer le projet d'analyse multiscalaire sur plusieurs décennies. L'objectif d'analyse des conséquences biographiques de la répression tenant compte de différents niveaux d'analyse et de la pluralité des sphères de vie des militant-es a conduit à la multiplication des sources susceptibles d'en donner la vision la plus complète possible. La mobilisation des entretiens et des mémoires a ainsi été complétée par la recherche des archives disponibles et la prise en compte de la dimension spatiale des mobilisations et des trajectoires biographiques qui sont apparues centrales à mesure que progressaient l'enquête et l'analyse. Face aux limites propres à chacune des sources mobilisées, il a fallu travailler à recouper, coudre et coller ensemble des résultats obtenus séparément pour recomposer un paysage politique, des groupes militants et des trajectoires individuelles tourmentées comme l'a montré la dernière partie de ce texte. Cette «triangulation » des méthodes est en réalité le produit de l'enquête, dans ses heurs comme dans ses malheurs. Au final, les difficultés se sont révélées productives car elles ont permis d'investiguer de nouvelles sources, telles les mémoires, qui n'étaient pas envisagées au départ.

\section{Déclaration de conflits d'intérêts}

L'auteur déclare n'avoir aucun conflit d'intérêt potentiel pour tout ce qui concerne le déroulement de la recherche, les droits d'auteur et/ou la publication de cet article.

\section{Financement}

L'auteur n'a bénéficié d'aucun soutien financier particulier relatif au déroulement de la recherche, à ses droits d'auteur et/ou à la publication de cet article.

\section{Remerciements}

Merci à Camille Hamidi qui a accompagné la maturation de ce texte, à Sophie Duchesne et Viviane Le Hay qui l'ont soutenu infatigablement ainsi qu'aux relecteur-trices anonymes de la première version. 


\section{Références}

Akal, E (2011) Kızıl feministler. Bir sözlü tarih çalışması (Les féministes rouges. Un travail d'histoire orale). Istanbul: Iletişim.

Barbot, J (2010) Mener un entretien de face à face. In Paugam, S (éd.) L'enquête sociologique. Paris : PUF, 115-141.

Baykam, B (2002) 68'li Yıllar. Eylemciler (Les années 68. Les activistes). Istanbul: Imge Kitabevi Yayınları.

Becker, J-J (1987) Le handicap de l'a posteriori. Questions à l'Histoire orale, Cahiers de l'IHTP $4: 95-99$.

Boumaza, M et Campana, A (2007) Enquêter en milieu «difficile». Revue Française de Science Politique 57/1 : 5-25.

Bourdieu, P (1986) L'illusion biographique. Actes de la recherche en sciences sociales 62-63 : 69-72.

Broadhead, R S et Rist, R C (1976) Gatekeepers and the Social Control of Social Research. Social Problems 23/3: 325-336.

Bué, N (2010) Gérer les relations d'enquête en terrains imbriqués. Risque d'enclicage et distances aux enquêtés dans une recherche sur une coalition partisane locale. Revue internationale de politique comparée 17/4 : 77-91.

Cefaï, D et Amiraux, V (2002) Les risques du métier. Engagements problématiques en sciences sociales. Partie 1. Cultures \& Conflits 47, URL : https://journals.openedition.org/conflits/829

Cemal, H (2012) 12 Eylül Günlügü. T. 1 Tank Sesiyle Uyanmak (Journal du 12 septembre.

Tome 1 : Se réveiller au son des chars). Istanbul : Everest Yayınları.

Collectif (2011) Kaktüsler Susuz da Yaşar. Kadınlar Mamak Cezaevini Anlatıyor (Les cactus vivent aussi sans eau. Les femmes racontent la prison de Mamak). Ankara : Dipnot.

Cormier, $\mathrm{P}$ (2016a) Les conséquences biographiques de l'engagement en contexte répressif. Militer au sein de la gauche radicale en Turquie : 1974-2014. Thèse de doctorat en science politique : Sciences Po Bordeaux/Université de Lausanne.

Cormier, P (2016b) De nouveaux horizons dans l'analyse des mouvements sociaux. À propos de mobilisations au Mali, au Maroc et en Turquie. Politix 112 : 187-196.

Cormier, P (2019) Reconstituer en politiste la géographie de mobilisations passées : quelques enjeux méthodologiques à partir de la gauche radicale turque dans les années 1970 à Ankara.

Carnets de géographes 12, URL : https://journals.openedition.org/cdg/4174

Cumhuriyet Ansiklopedisi (Encyclopédie de la République) (2002) Cilt 3 (1961-1980) [Tome 3 (1961-1980)]. Istanbul : Yapı Kredi Yayınları.

Cumhuriyet Ansiklopedisi (Encyclopédie de la République) (2002) Cilt 4 (1981-2000) [Tome 4 (1981-2000]. Istanbul : Yap1 Kredi Yayınları.

Curtis, R L et Zurcher, L A (1973) Stable Resources of Protest Movements: The MultiOrganizational Field. Social Forces 52/1: 53-61.

Darmon, M (2005) Le psychiatre, la sociologue et la boulangère : analyse d'un refus de terrain. Genèses 58 : 98-112.

de Castro Rocha, D et Goirand, C (2010) L'accès aux archives du Parti des travailleurs au Brésil : expériences croisées et jeux d'échelles. Revue internationale de politique comparée 17/4 : 109-125.

Delikanlı, E et Delikanlı, Ö (2014) Keşke Bir Öpüp Koklasaydım. Geride Kalan Aileler 12 Eylül'ü Anlatıyor (Si seulement je pouvais sentir un baiser. Les familles restées à l'arrière racontent le 12 septembr)]. Istanbul : Ayrint1.

Denave, S (2015) Reconstruire sa vie professionnelle. Sociologie des bifurcations biographiques. Paris, PUF. 
Dev-Genç (1999 [1988]) Bă̆ımsızlık, demokrasi ve sosyalizm mücadelesinde. Gençlik. Cilt. 1 1974-1980 (Dans la lutte pour l'indépendance, la démocratie et le socialisme. La jeunesse. Tome 1 : 1974-1980). Istanbul : Boran Yayınevi.

Dorronsoro, G (2005) Mobilisations et régime sécuritaire. In Dorronsoro, G (éd.) La Turquie conteste. Mobilisations sociales et régime sécuritaire. Paris : CNRS Éditions, 13-30.

Duchesne, S (2008) La Démocratie de l'abstention, Céline Braconnier et Jean-Yves Dormagen. Revue internationale de politique comparée 15/4 : 705-712.

Fillieule, O (2007) On n'y voit rien. Le recours aux sources de presse pour l'analyse des mobilisations protestataires. In Favre, $\mathrm{P}$ et alii. L'atelier du politiste. Paris : La Découverte, 215240.

Fillieule, $\mathrm{O}$ et alii (éds.) (2018) Changer le monde, changer sa vie. Enquête sur les militantes et les militants des années 68 en France. Arles : Actes-Sud.

Fournier, P (1996) Des observations sous surveillance. Genèses 24 : 103-119.

Freedman, D et alii (1988) The life-history Calendar: A Technique for Collecting Retrospective Data. Sociological Methodology 18: 37-68.

Gehver, D (2014) Mamak. Ardından tavşan yaşamı (Mamak. Après ma vie de lapin). Istanbul : Belge.

Ginzburg, C (1980) Signes, traces, pistes. Racines d'un paradigme de l'indice. Le Débat $6: 3$ 44.

Gourisse, B (2011) Variation des ressources collectives et organisation des activités de violence au sein du Mouvement nationaliste en Turquie (1975-1980). Cultures \& Conflits 81-82:81100.

Gourisse, B (2014) L'Etat en jeu. La violence politique en Turquie (1975-1980). Paris : Karthala.

Grojean, O (2010) Les aléas d'un terrain comme révélateurs de sa structuration. Gestion et objectivation d'une relation d'enquête sur une mouvance radicale et transnationale. Revue internationale de politique comparée 17/4 : 63-76.

Grossetti, M (2006) L'imprévisibilité dans les parcours sociaux. Cahiers internationaux de sociologie 120/1 : 5-28.

Lahire, B (2001) L'homme pluriel. Les ressorts de l'action. Paris : Nathan.

Lahire, B (2012) Monde pluriel. Penser l'unité des sciences sociales. Paris : Seuil.

Leclercq, C (2005) «Raisons de sortir». Les militants du Parti communiste français. In Fillieule, $\mathrm{O}$ (éd.) Le désengagement militant. Paris : Belin, 131-154.

Lesure, M (2013) Lépante. La crise de l'Empire ottoman. Paris : Gallimard.

Massicard, E (2002) Etre pris par le mouvement. Parties 1 \& 2. Cultures et Conflits 47 URL : http://conflits.revues.org/index $840 . h t m l$

Massicard, E (2012) Répression et changement des formes de militantisme : carrières et remobilisation à gauche après 1980 en Turquie. European Journal of Turkish Studies 15, URL : https://ejts.revues.org/4691

McAdam, D (2012) Freedom Summer. Luttes pour les droits civiques Mississipi 1964. Marseille : Agone.

Müftüoğlu, O (1989) Devrimci Yol Savunmast. 12 Eylül ve Türkiye Gerçeği (Défense de Devrimci Yol. La vérité sur le 12 septembre et la Turquie). Istanbul : Bir Gün.

Neveu, E (2008) Trajectoires de « soixante-huitards ordinaires ». In Damamme, D et alii. (éds.) Mai-juin 68. Paris: Editions de l'Atelier, 306-318.

Norton Cru, J (2008) Du témoignage. Paris : Allia.

Offerlé, M (1987) Les partis politiques. Paris : PUF.

Osa, M (2003) Solidarity and Contention: Networks of Polish Opposition. Minneapolis : University of Minnesota Press. 
Pagis, J (2014) Mai 68, un pavé dans leur histoire. Evènements et socialisation politique. Paris : Presses de Sciences Po.

Passy, F (1998) L'action altruiste. Genève : Droz.

Passy, F (2005) Interaction sociales et imbrications des sphères de vie. In Fillieule, O (éd.) Le désengagement militant. Paris : Belin, 111-130.

Pennetier, C et Pudal, B (éds.) (2002) Autobiographies, autocritiques, aveux dans le monde communiste. Paris : Belin.

Revel, J (éd.) (1998) Jeux d'échelles. La micro-analyse à l'expérience. Paris : Gallimard-Seuil. Romani, V (2007) Enquêter dans les Territoires Palestiniens. Comprendre un quotidien au-delà de la violence immédiate. Revue Française de Science Politique 57/1 : 27-45.

Sağır, A (2015) Bizi Güneşe Clkardılar (Ils nous ont emmenées au soleil). Istanbul : Ayrıntı.

Schwartz, O (1990) Le baroque des biographies. Cahiers de philosophie 10 : 173-183.

Smith, S et Watson, J (2010) Reading Autobiography: A Guide for Interpreting Life Narratives. Minneapolis: University of Minnesota Press.

Stavo-Debauge, J, Roca i Escoda, M et Hummel, C (2017) Enquêter. Rater. Enquêter encore.

Rater encore. Rater mieux. SociologieS, URL:

https://journals.openedition.org/sociologies/6084

TMMOB Mimarlar Odası Ankara Şubesi [Chambre des architectes, succursale d'Ankara] (2007) Tanıkların Ulucanlar'ı. sözlü tarih (Témoins d'Ulucanlar. Histoire orale). Ankara : TMMOB.

Vannetzel, M (2010) À la frontière du parti : jeux d'inclusion et d'exclusion d'une chercheuse chez les Frères musulmans égyptiens. Revue internationale de politique comparée 17/4 : 4762.

Zileli, G (2002) Havariler (1972-1983) (Les apostats (1972-1983)). Istanbul : Iletişim.

Zileli, G (2003) Sapak (1983-1992) (Détour (1983-1992)). Istanbul : Iletişim. 